\section{New PPE mask range begins killing SARS-CoV-2 on contact}

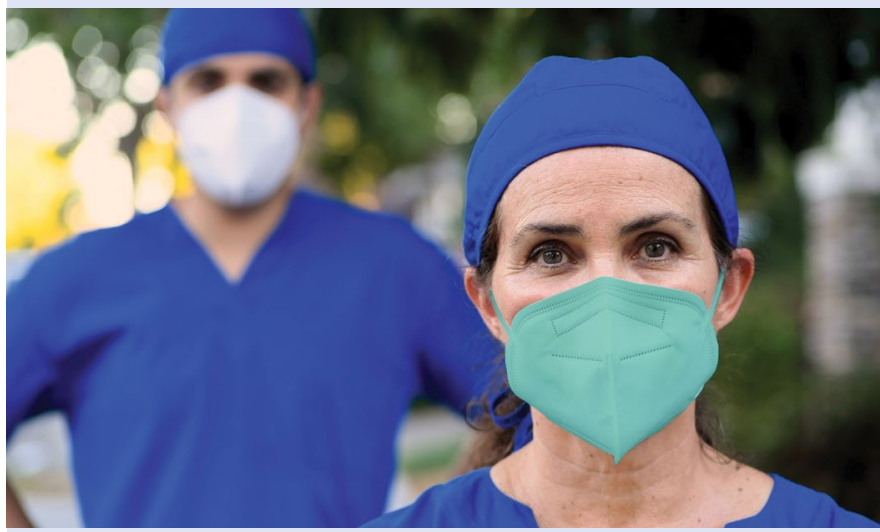

Lancaster-based ViraCoat Limited has announced the global launch of ViraCoat 3 Ply Type 2R Surgical Face Mask, ViraCoat FFP2 Respirator Mask and ViraCoat FFP3 Respirator Mask, a new range of antiviral and antimicrobial PPE masks that begin to kill SARS$\mathrm{CoV}-2$ and other viruses on contact. This is a major breakthrough in reducing risks, to staff and patients, of COVID-19. The product has gone through rigorous validation with Lancaster University and spent a year in development, and is now going into production in the UK.

The ViraCoat masks are impregnated with a novel and patented antiviral and antimicrobial coating that completely kills SARS$\mathrm{CoV}-2$ virus within minutes. The entire surface of the mask is covered in the antimicrobial coating, including the straps; this significantly reduces the risk of contamination during handling of the masks and therefore transfer of the virus to the wearer or patient, even if the mask is removed and replaced several times during a shift. Many hospitals report health workers changing masks between three and eight times throughout the course of the day as they move between patients or tasks. ViraCoat's COVID-19-eliminating masks could save millions of tons of masks ending up in landfill and our oceans every year.

The masks are ergonomically shaped for comfort and have an adjustable nose bridge for an optimal seal to prevent contamination by infectious aerosols. They are a distinctive green colour to easily differentiate from standard PPE. At this stage, the ViraCoat antimicrobial coating is being applied to masks conforming to the filter-barrier requirements of EU Standard EN149:2001 + A1: 2009 type filtering efficiency standard to meet current regulations. However, the coating obviates the need for these types of barriers and new standards are being sought that will allow ViraCoat antimicrobial masks to be a lighter, more comfortable single layer, which can be repeatedly washed or sterilised.

ViraCoat is a subsidiary of ViraCorp, a group of biomedical research and development companies focused on developing viral defence products against the viral outbreaks, pandemics and endemics that threaten world health; the ViraCoat range of masks is the first product to go into production. The masks will be in UK production at scale in October with the highest standards of quality control.

https://viracorp.global/

\section{Value, strength and beauty that lasts}

\section{COLTENE's BRILLIANT COMPONEER is \\ a direct composite veneering system that proves stunning, enduring results can be delivered cost-effectively. \\ BRILLIANT \\ COMPONEER \\ is comprised of \\ prefabricated, pre-shaped \\ shells for chairside}

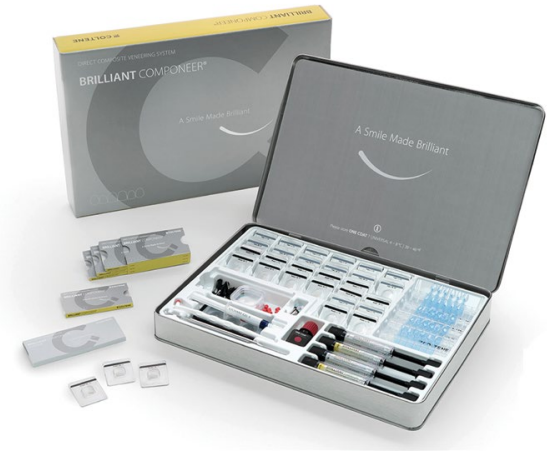

treatments. It might be innovative - the material utilises sub-micron filler technology - but it's an easy-to-use system too.

In one session, you will create a beautiful smile. This means value for you and your patient, but aesthetics won't be compromised as BRILLIANT COMPONEER also offers exceptional polishability and long-lasting gloss.

Work conservatively and meet your patients' expectations every time. Join other great dentists in using BRILLIANT COMPONEER, from COLTENE.

For more on COLTENE, visit www.coltene.com, email info.uk@ coltene.com or call 08002545115.

\section{Truly one of a kind}

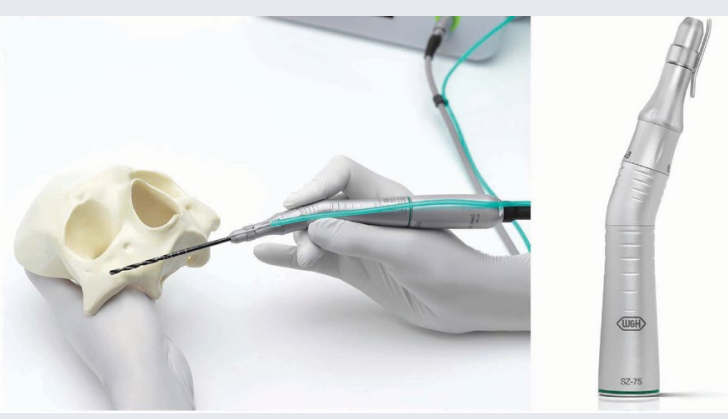

Looking for a safe and effective surgical handpiece for zygomatic implant placement? Consider the SZ-75 Zygoma handpiece from W\&H. Developed in collaboration with leading implant dentists, this 20:1 handpiece is truly one of a kind - especially when treating patients with complex clinical needs.

Its unique contra-angle allows for optimal access to difficult areas of the zygomatic bone, ensuring it's safe to use and consistently accurate. No other handpiece can be used at this angle, but the SZ-75 from W\&H makes it look easy.

Unlike conventional surgical handpieces, the SZ-75 is designed for use with Zygoma burs.

Using its technology, the Zygoma handpiece removes the need for manual cooling with an external spray, ensuring the treatment site is cooled with utmost efficiency.

If you're looking for a unique instrument to enhance your zygomatic implant workflow then find out more today.

To find out more visit www.wh.com/en_uk, call 01727874990 or email office.uk@wh.com. 